\section{The ATLAS Facility at Argonne National Laboratory}

RECEIVED

JUL 291997

OSTI

\section{Some Historical Remarks}

The Argonne Tandem Linac Accelerator System (ATLAS) is a superconducting low-energy heavyion accelerator [1]. Its primary purpose is to provide beams for research in nuclear structüre physics. It therefore seems quite fitting that ATLAS is located in Building 203 at Argonne National Laboratory, the same building where Marie Goeppert-Mayer worked on her Nobel-prize winning contributions to the nuclear shell model.

ATLAS grew out of the nuclear physics program with the tandems purchased by the Argonne Physics Division from High Voltage Engineering Corporation in the 1960s: the EN Tandem which was installed in 1960/1961, and the present $F N$ tandem which replaced the EN tandem in 1967. During the 1960 s the tandem was primarily used with light ion beams. During that period, reaction spectroscopy at
Argonne helped lay many of the cormer stones in our understanding of the structure of the nucleus. This included single-particle levels, nucleon-nucleon two body effective interactions, and other microscopic aspects of nuclear structure and dynamics. In the early 1970 s light heavy-ion beams became available and a program of nuclear reactions and structure studies with heavy ions started. It soon became obvious that it would be desirable to upgrade the facility to a higher voltage, in order to allow acceleration of heavier nuclei to energies above the Coulomb barrier.

In the early 1970s development work started at Argonne on a superconducting post-accelerator [2]. The effort initially focused on niobium helix structures, but soon was redirected towards the mechanically more stable split-ring resonator [3] based on the early developments at Caltech. The superconducting res- onator development at Argonne resulted in major advances. Since niobium metal possesses excellent superconducting properties but is a poor heat conductor, resonators were developed from explosively bonded niobium-copper composite metals, with copper providing the cooling path. Control of the high-Q resonators was achieved with a voltage controlled reactance (VCX). Adequate cooling was established with a robust liquid-helium continuous flow system. Many other innovative solutions to technical problems in superconducting RF acceleration, cryogenics, and accelerator beam optics were developed. In 1978, the first superconducting accelerator for ions was brought into operation at Argonne. The concept of independently phased resonators, made feasible through the low power levels in the high-Q superconducting cavities, provided for new aspects of accelerator design and operation. The independent phasing allows matching sequential resonators to a broad velocity profile thus permitting flexible acceleration of a broad range of ion species. It also allowed incremental use of sections of resonators for experiments when completed. This was crucial for the ability to construct a useful post-accelerator (the 'booster') since funding was provided as R\&D funds in incremental steps on a yearly basis.

Figure 1. Layout of the ATLAS Facility

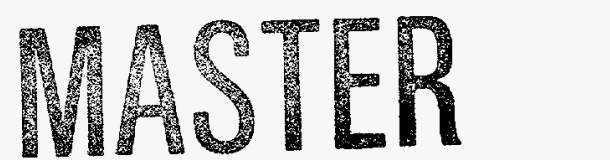




\section{DISCLAIMER}

This report was prepared as an account of work sponsored by an agency of the United States Government. Neither the United States Government nor any agency thereof, nor any of their employees, make any warranty, express or implied, or assumes any legal liability or responsibility for the accuracy, completeness, or usefulness of any information, apparatus, product, or process disclosed, or represents that its use would not infringe privately owned rights. Reference herein to any specific commercial product, process, or service by trade name, trademark, manufacturer, or otherwise does not necessarily constitute or imply its endorsement, recommendation, or favoring by the United States Government or any agency thereof. The views and opinions of authors expressed herein do not necessarily state or reflect those of the United States Government or any agency thereof. 


\section{DISCLAMMER}

Portions of this document may be illegible in electronic image products. Images are produced from the best available original document. 


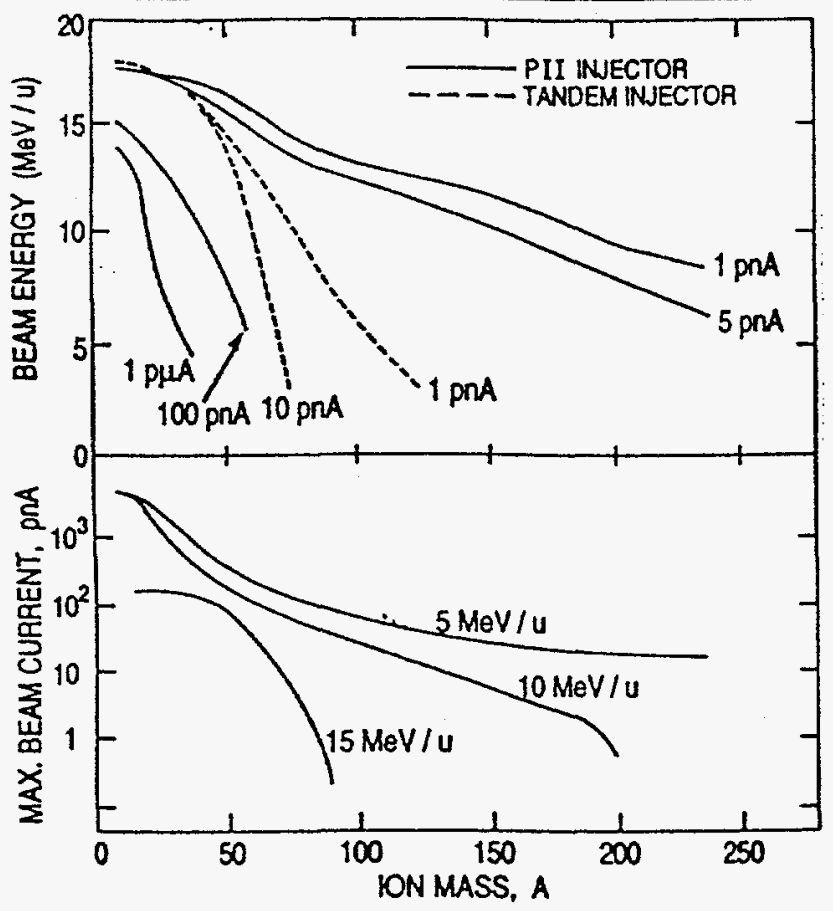

Figure 2. Energies and intensities for various beam species at ATLAS.

Major milestones in the history of the ATLAS Facility were: the on-line operation of the first resonator and booster section for research in 1978; the completion of the booster linac with 24 splitring resonators in 1982 (at that time already about 10,000 hours of beam on target had been logged with a constantly changing (and growing) post-accelerator), completion and first acceleration of beams through the full ATLAS system in 1985; and completio of the positive ion injector PII in 1992 [4]. The last step concluded the creation of the state-of-the-ar low-energy heavy ion accelerator for nuclear structure research.

\section{The Present Facility}

ATLAS now provides ion beams with energies above the Coulomb barrier for all stable isotopes and elements up to uranium, and with excellent beam quality: $100 \%$ duty cycle, small transverse and longitudinal emittances, subnanosecond timing, casy energy variability, and rather intense beams even for low-abundance isotopes.
The cutaway in Figure 1 shows the layout of the present facility with the two injectors (the FN tandem and the positive ion injector PII), the booster linac, and the ATLAS linac additions. The target areas house a variety of experimental equipment. This accelerator system provides an acceleration voltage of about 50MV. With the bigh charge state ions from the ECR ion source (or after energy heavy-ion research. Because of the significance of PII we discuss here some relevant details (Figure 4). It consists of an electron cyclotron-resonance ion source (ECR) on a $300-\mathrm{kV}$ platform coupled to a 12-MV superconducting drift-tube-type linac. This system represents the first use of an ECR source at high voltage and the first application of superconducting resonators for the acceleration of very low velocity $(B \geq 0.008)$ ions.

The injector linac is formed by an array of 18 superconducting niobium 4-gap structures that are independently phased. Four different types (sizes) of structures are needed to accelerate the heaviest particles $(q / A=0.10)$ from $B \approx$ 0.008 to $B \geq 0.043$. These structures, of which three classes operate at an RF frequency of $\mathbf{4 8 . 5}$ stripping from the FN tandem), all ions up to uranium can be accelerated to energies well above the Coulomb barrier. Intensities and energies for various beam species are shown in Figure 2. Figure 3 illustrates the impact of the ECR ion source and PII injector linac on the distribution of ion beams used in experiments: in 1991 , before this upgrade, beam species concentrated below mass 60 while two years later, with PII operational, a large fraction of beams used in experiments have mass 100 to 238.

\section{The PII Injector}

As mentioned above, completion of the positive ion injector (PII) was crucial for the creation of what is probably the state-of-theart accelcrator for low.
ATLAS BEAMS FOR 1991

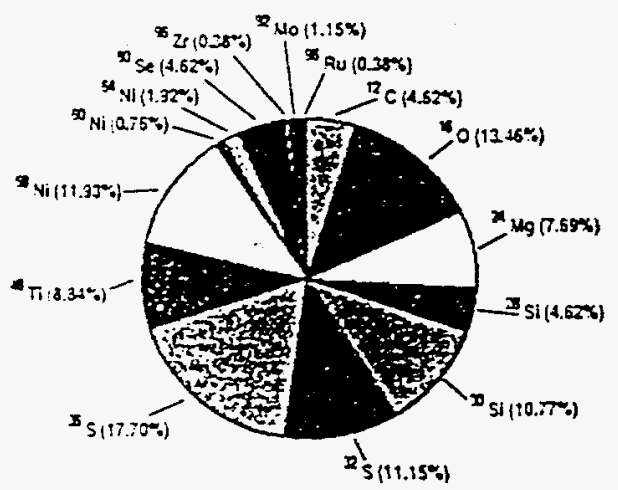

ATLAS BEAMS FOR 1993

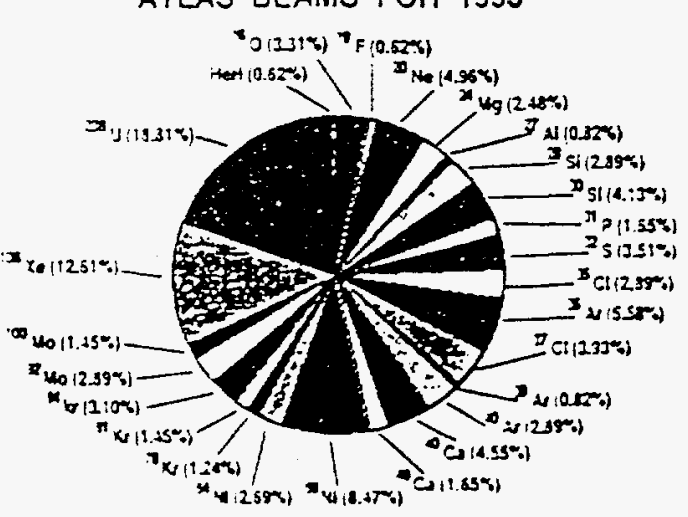

Figure 3. Distribution of ion beams at ATLAS in 1991 und 1993 ibefore und after completion of the posutue wan injector Pll) 


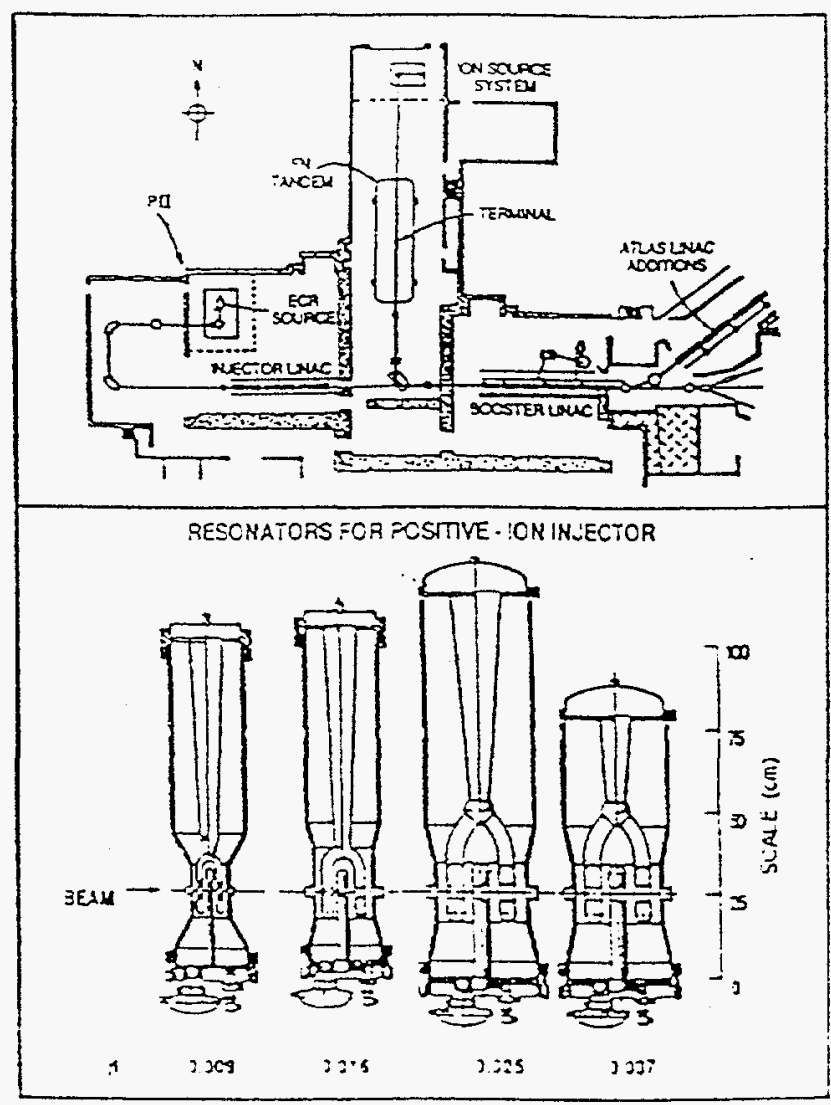

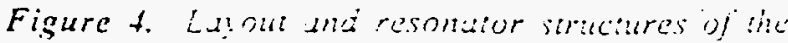
positive ion injector Pll at .tTL.AS lubovel. Photogrupin of a Pll resonutor section holowl.
M Hz. make use of :cchnology developed for the main part of the ATLAS linac, including housings made of a composite material consisting of niobium explosively bonded to copper.

The main technical challenges for PII were (a) to bunch the very slow-moving ions from the source into pulses $<0.3$ ns wide at the first accelerating structure, (b) to accelerate these ions without much degradation of beam quality, (c) to avoid loss of beam because of defocusn' vaused by RF acceleration at low velocity, and (d) to achieve phase conirol of the res. unators. It rumed vut that all of these

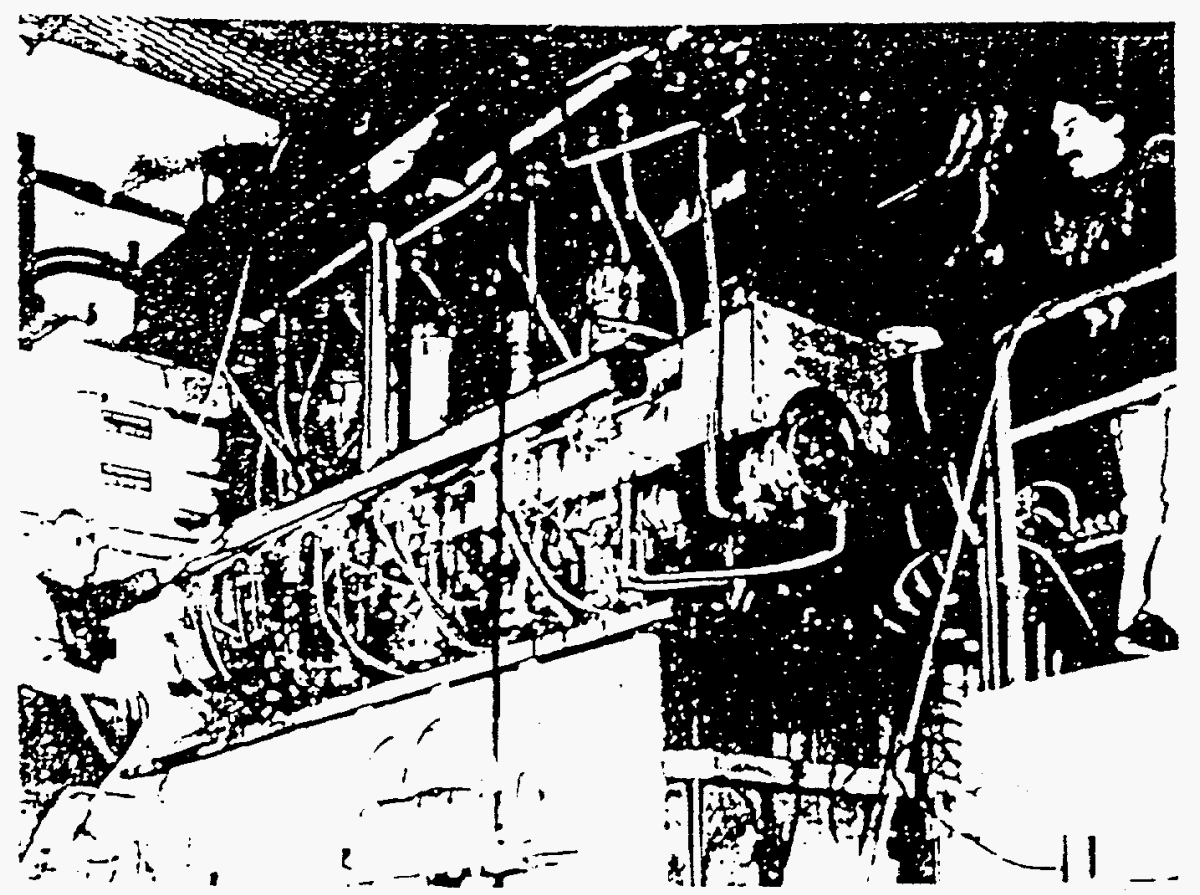

problems were solvable. About $60 \%$ of the initial beam is bunched into $12.125-\mathrm{MHz}$ pulses $0.3 \mathrm{~ns}$ wide by a 2-stage system consisting of a 4-harmonic buncher on the voltage platform $(B=0.0015)$ followed by a $24.25-\mathrm{MHz}$ buncher at the entrance to the linac $(B=$ 0.008 ). The effects of transverse defocusing are controlled in the difficult low-velocity end of the linac by a superconducting solenoid after each accelerating structure, by alternating-phase focusing in the first structure, and by velocity focusing caused by the extremely rapid increase in velocity in the first few structures. The result is $-100 \%$ beam transmission. Similarly, the compact geometry employed leads naturally to the preservation of longitudinal beam quality if the linac is properly tuned; indeed, the longitudinal emittance for the beam from PII is only about $20 \pi \mathrm{keV}$-ns even for the heaviest ans, a new standard of excellence.

Superconducting RF and Linac Development

There is an ongoing program at ATLAS in basic research and development of the accelerator physics of superconducting linacs and elated echnologtes. Wuch or this effort is rolated Jirectly to uperading or improving :echnology of ATLAS. with a continuing goal of enhancing its capabilites. This inc!udes RiD related to the :opics of superconducting accelerator struceursi dectron equletron rescnance son sources. acculerator

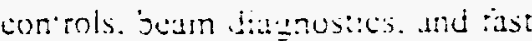
an:ere echnologe:

Some uf hase acti:tates aro generic researcti in the bisk

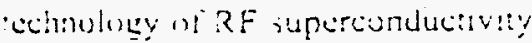
w:h emphusis un low-trequency structures used tor heidy-tons and lo:v relocit:es Subjects

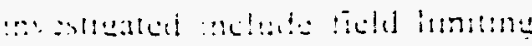

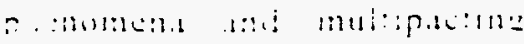

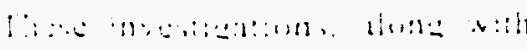

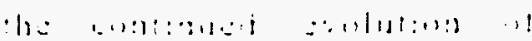




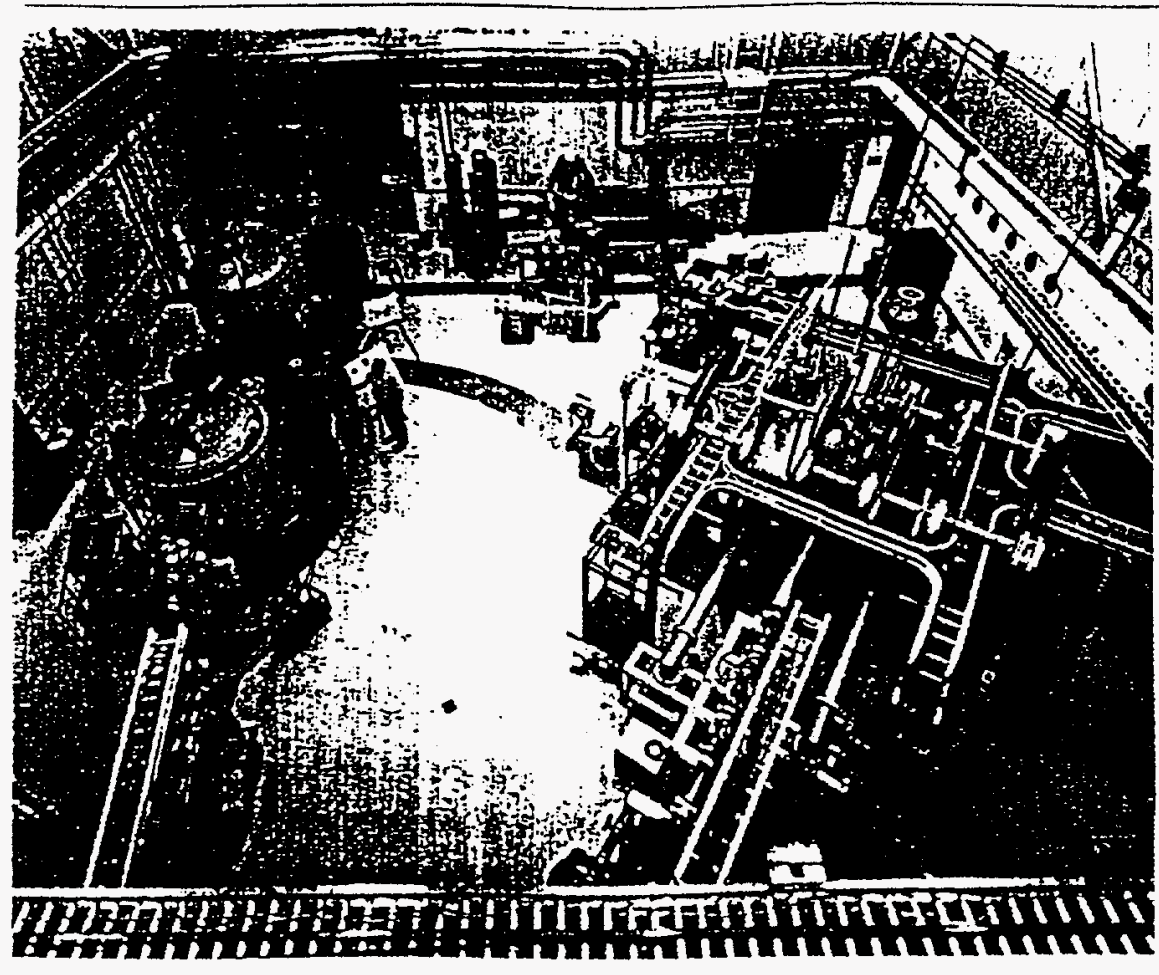

Figure 5. Target area $I V$ at ATLAS with the Fragment Mass Analyzer (FMA) on the left, and the ATLAS Positron Experiment (APEX) on the right.

fabrication methods, should lead to more cost-effective structures with increased performance. Some progress was made recently towards observing and controlling the Qdegradation effect from hydrite formation in niobium resonator structures.

A prototype cylindrically symmetric quarter-wave coupled linac structure was constructed as part of the joint project with the Nuclear Science Centre in Delhi, India. The construction of a niobium superconducting radiofrequency quadrupole (RFQ) structure is nearing completion in collaboration with Acc Sys Technology Inc. Production of superconducting cavities for the University of Sao Paulo, Brazil, is also nearly complete. Altogether resonators of the ATLAS type have been fabricated at Argonne and provided for accelerator upgrades at Florida State University. Kansas State University, the University of Sao Paulo in Brazil, and currently the Vuclear Seience Centre, India.
Detailed studies of the dynamics of the front-end of the PII linac are in progress with emphasis to possibly extemdomg the technology to even lower velocities. This work aims toward developing an optimal solution for the front-end of an ISOL celerator facility.

Some effort is also directed towards improving ECR ion source performance as well as developing techniques which will apply to increasing charge states for future radioactive beam accelerators. A sputter method to generate beams from solids in an ECR ion source was developed.

Construction of an advanced ECR ion type radioactive ac- source with a stronger magnetic field and higher operating frequency is underway in collaboration with the ECR group at LBL Berkeley, and completion of this second ECR source at ATLAS is scheduled for the spring of 1996.

\section{The Experimental Equipment}

ATLAS delivers beam to 12 beam lines and target stations which are equipped with a variety of experimental equipment (see Figures 1 and 15). In addition to general purpose beamlines and scattering chamber facilities, there are several pieces of major, in some cases unique, equipment. These include: Two Enge split-pole magnetic spectrographs. Originally built in the 1960s for experiments with light ions $\left(p, d,{ }^{3} \mathrm{He}\right.$, etc.), these spectrometers have intrinsic properties well-matched to reaction studies with heavy ions: good resolution $(\sim 1: 2000)$ and solid angle ( $\leq 5 \mathrm{msr}$ ); large momentum acceptance $(60 \%)$, of importance because of heavy-ion charge-state distributions; and momentum dispersion well-matched to state-of-

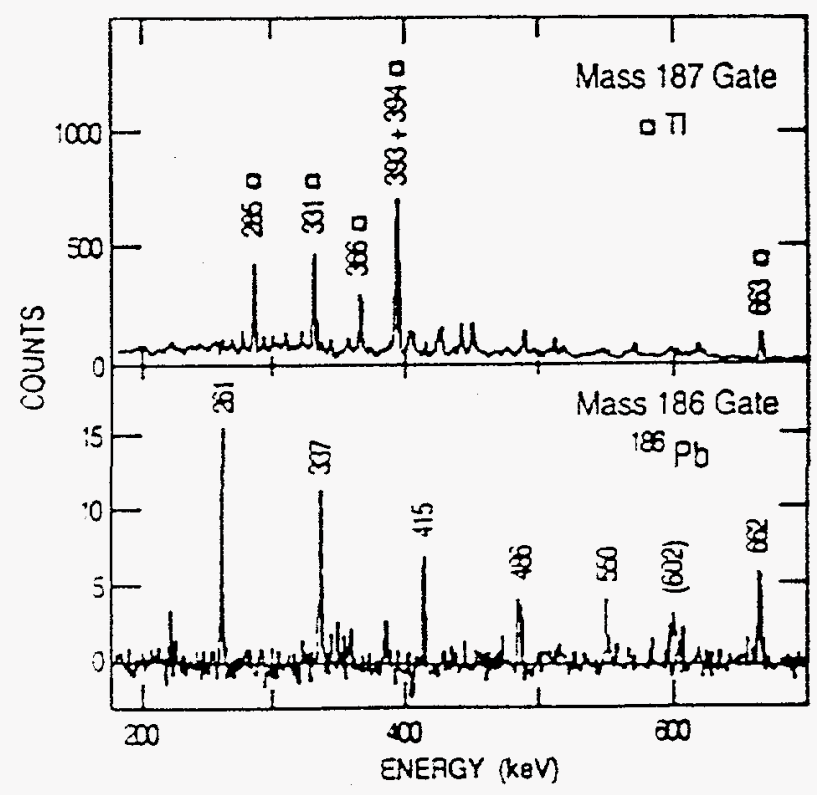

Figure 6. Gamma-ray spectra for $187 \mathrm{Tl}$ and $196 \mathrm{~Pb}$. selected by mass gates on evaporation residues analyzed by the F.V.A. 



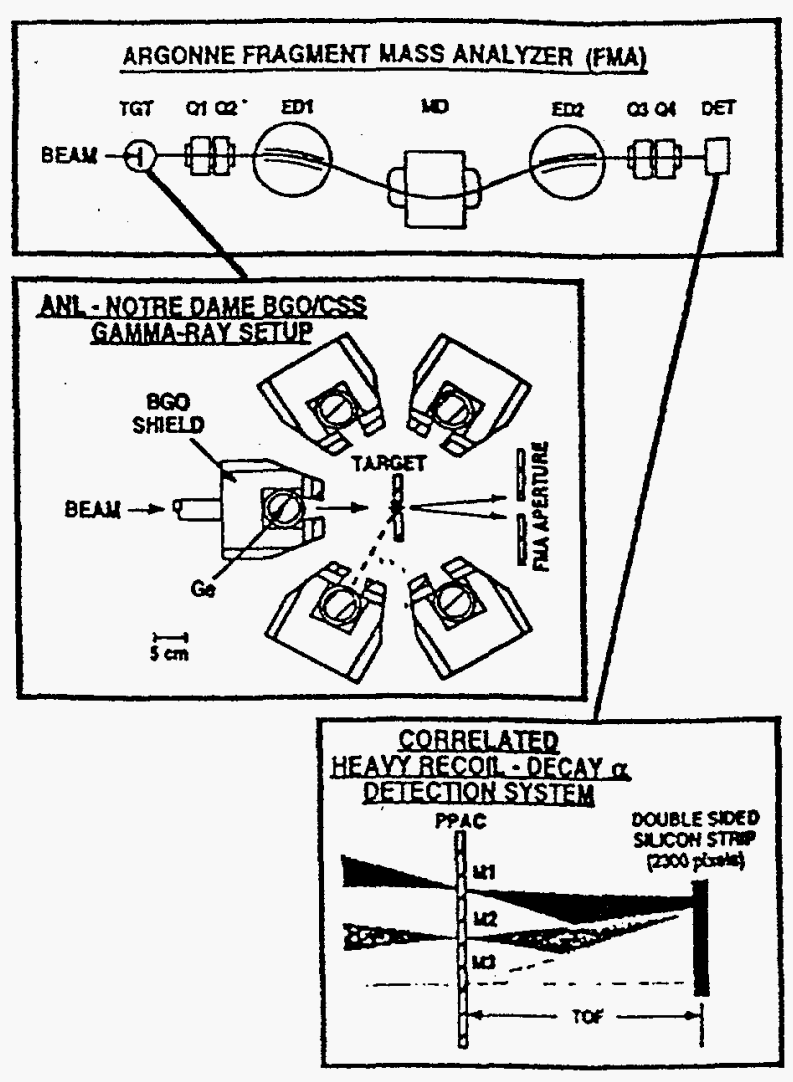

Figure 7. Schematic layout of FMA and its associated detector systems: a) for coincidences between gamma rays near the target and recoils, b) for delayed particle spectroscopy at the focal plane correlated with recoil implants.

the-art position detector resolution. The spectrometers are equipped with focal plane detectors for heavy ions providing good momentur resolution and excellent particle identification. One of the split- pole spectrographs is also equipped with devices for use as a gas-filled magnetic spectrometer for fusion reactions, AMS, and other applications which need very good isobar separation.

The Argonne/Notre Dame CSS/BGO gamma-ray facility: This facility consists of 12 Compton suppressed high-resolution germanium detectors and an energy-sum/multiplicity inner array, with $80 \%$ solid angle coverage, consisting of 50 hexagonal BGO crystals. The system is used in stand-alone mode for high spin studies, in particular superdeformation, but also in recoil tagging mode in front or near the focal plane of the FMA.

The Fragment Mass Analyzer (FMA): The FMA (Figure 5) is a recoil mass spectrometer that came into operation in 1991. It consists of electric and magnetic ion optical elements that provide excellent mass resolution for heavy fragments (> 500 has been achieved for heavy nuclei) [5]. The FMA is normally positioned at 0 degrees but it can be moved out to scattering angles of 30 degrees. With its excellent properties, including large solid-angle and momentum acceptances, the FMA is currently used in about $40 \%$ of all ATLAS experiments.

The Lepton-Spectrometer APEX (Figure 5): A large solidangle (nearly $4 \pi$ ) two arm electronpositron spectrometer with solenoidal magnetic guide field and two arrays of about 200 high-resolution Si-detectors each was recently constructed by a collaboration involving 7 universities and Argonne for detection of leptons and lepton pairs in the range $150 \mathrm{keV}$ to 1 $\mathrm{MeV}$. In addition, this spectrometer is equipped with large solid-angle heavy ion detectors covering most of the forward hemisphere. High resolution germanium detectors can be positioned near the target to observe gamma rays.

The superconducting electron spectrometer: A large solid-angle spectrometer exists to efficiently collect conversion electrons on a highresolution $\mathrm{Si}$ detector. This device can also be used successfully in measurements of $B$ decay spectra (e.g. the $17 \mathrm{keV}$ neutrino experiment).

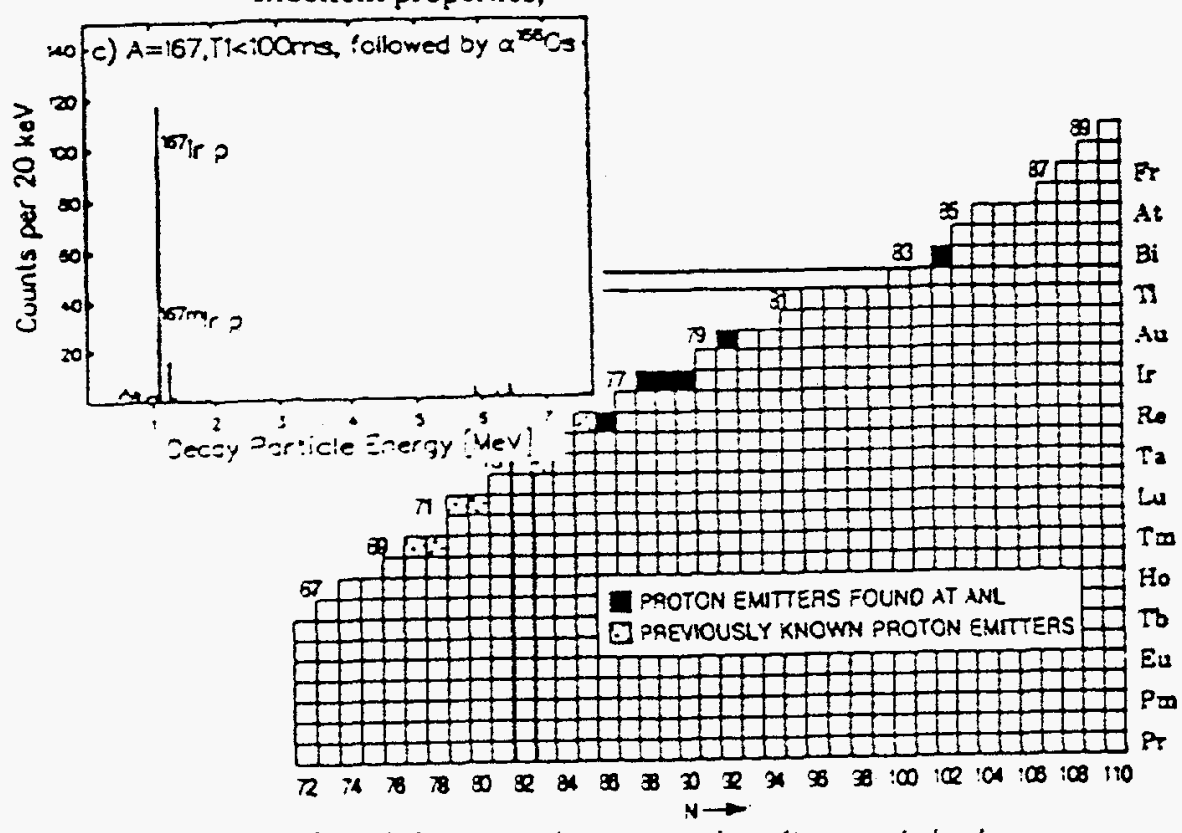

Figure 8. Churt of nuclides near the proton drip line and the heavy procon emiticrs recently discovered at the F.MA 


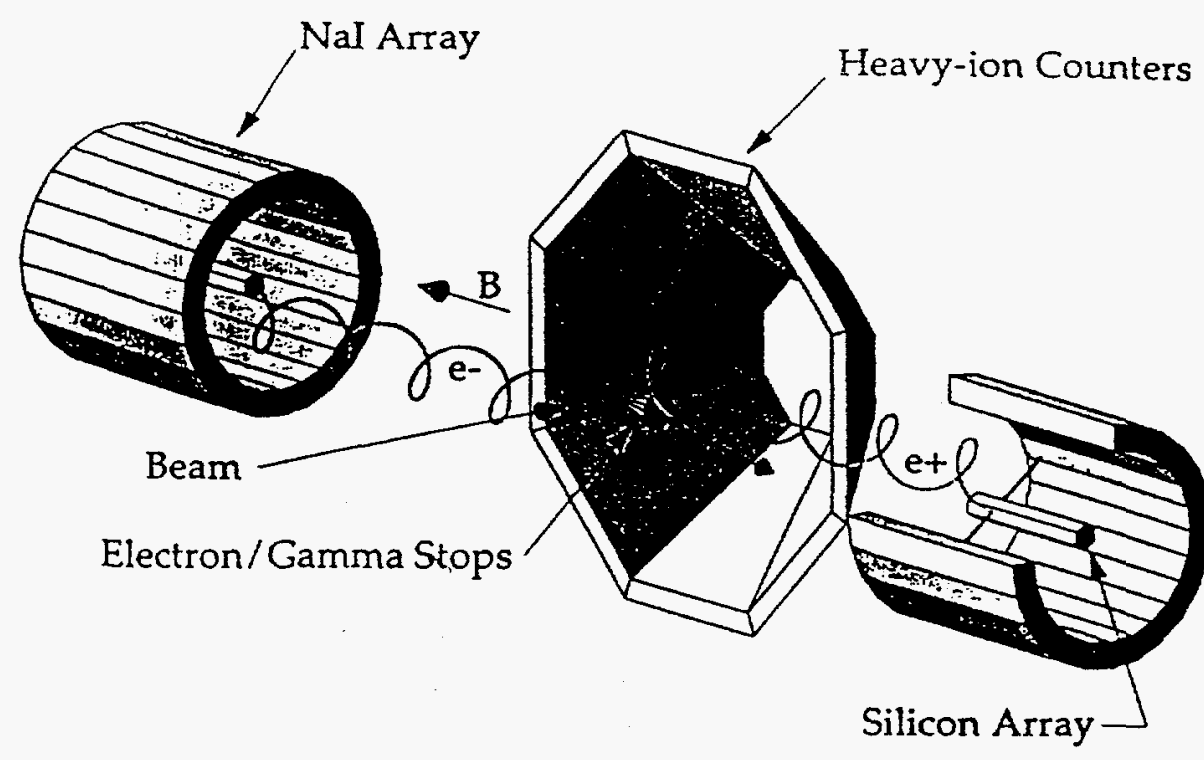

Figure 9. Schematic of the APEX electron-positron coincidence set-up at ATLAS.

The Research Program

The current research program is largely focused on aspects of nuclear structure and dynamics and on the interplay between the two. In addition, programs exist in atomic physics with highly-stripped ions, in material sciences, and in some applied research. In the following we discuss some recent examples.

Gamma-ray spectroscopy of high-spin states has been a major program over the last decade at ATLAS. The program has focused on superdeformation, in particular in the mass 190 region where its occurrence was first observed at Argonne. These studies were mostly carried out at the Argonne/Notre Dame CSS/BGO setup described above. Recently, the FMA has been employed to tag weak reaction channels in coincidence with gamma rays. Especially for heavy nuclei, fission is the dominant decay channel, and clean gamma-ray spectra from compound nucleus-like recoils are difficult to measure. Tagging with the FMA substantially cleans up the gammaray spectra and provides mass identification of the heavy residue. This is illustrated in Figure 6 with the example of $186 \mathrm{~Pb}$ [6]. The very neutron-deficient $\mathrm{Pb}$ isotopes are of much current interest because they exhibit shape coexistence between a spherical groundstate and a deformed prolate excited configuration located very low in excitation energy. The results of this work confirm and extend a band of levels tentatively proposed earlier and provide a definitive mass assignment for the nucleus emitting the observed gamma rays. The band observed in $186 \mathrm{~Pb}$ bears a very close resemblance to the isotone $184 \mathrm{Hg}$. supporting the view that the $186 \mathrm{pb}$ band is built upon a prolate structure.

A broad spectrum of experiments are being performed at the FMA. Figure 7 shows the schematic layout of the detector systems yrast band in the used, either for prompt gamma-ray spectroscopy in front of the FMA or for delayed, correlated particle spectroscopy after the FMA. The recent results on new spontaneous proton emitters are particularly intriguing [7]. With excellent mass resolution to identify and tag the recoiling compound nucleus (after particle evaporation), superior beam suppression (often better than 1 in $10^{12}$ ), and large acceptances in solid angle and momentum, clean proton-decay spectra have been recorded for five new proton emitters discovered at the FMA (Figure 8), the heaviest yet observed.

APEX is an experiment to study lepton production in collisions of very heavy ions. In these collisions electrons rapidly rearrange around the di-nuclear system and for a short time experience a combined nuclear charge so large that the inner electron shells dive into the negative energy continuum. The Coulomb field has enough energy for spontaneous electron-positron production. A K-shell vacancy could be filled by a produced electron and a positron emitted.

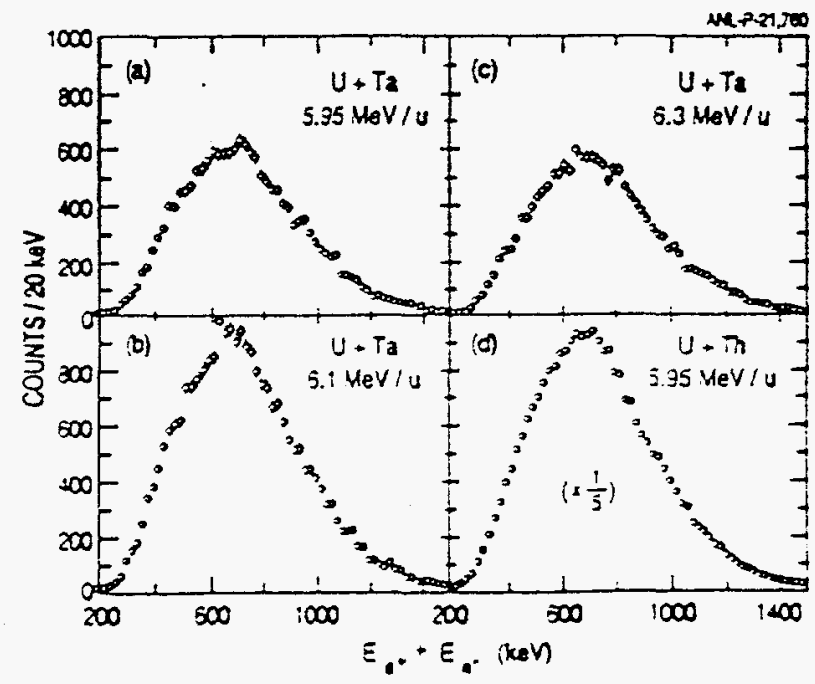

Figure 10. Electron-positron sum energy specira measured with APEX for the two collision sysiems $238 \mathrm{U}+181 \mathrm{Ta}$ and $238 \mathrm{U}-232 \mathrm{Th}$ 

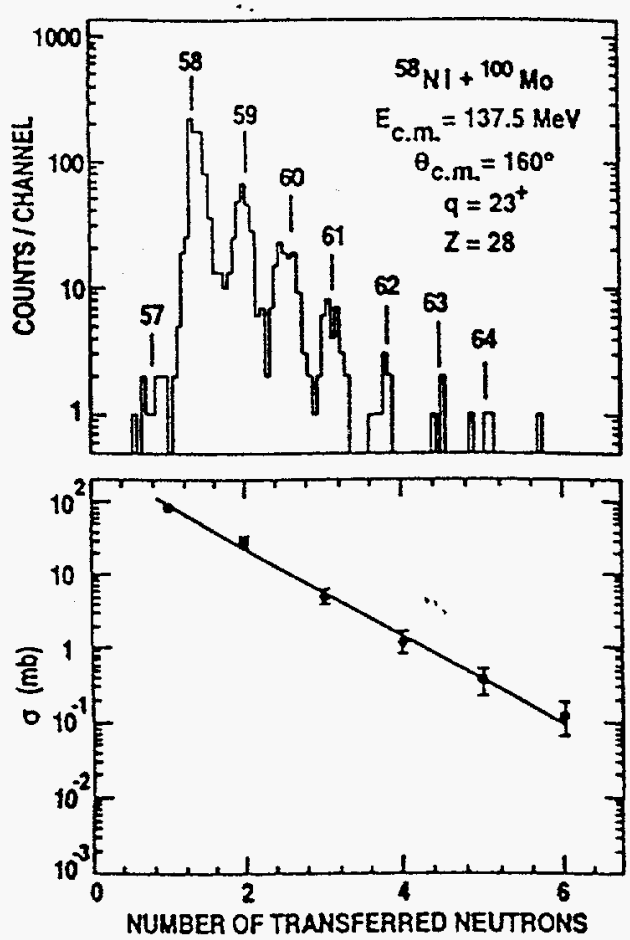

Figure 11. One- to six-neutron transfer cross sections measured for the $58 \mathrm{Ni}+100$ Wo system at sub-barrier energies

Realistic calculations set a time scale for the collision that produces a broad positron spectrum hundreds of $\mathrm{keV}$ wide. The observation of positron lines and of sharp electronpositron pair lines at GSI was thus completely unexpected. This prompted the APEX experiment. The setup is shown schematically in Figure 9. The first results from the collision systems ${ }^{238} \mathrm{U}+{ }^{181} \mathrm{Ta}$ and $238 \mathrm{U}-232 \mathrm{Th}$ are shown in Figure 10. With cross section limits well below the values reported by GSI, no sharp electronpositron pair lines have yet been observed. [8]

The range of ion beam species at ATLAS has allowed a broad range of reaction studies. An example is shown in Figure 11. Multiple-neutron transfers were studied with the Enge split-pole spectrograph. It was suggested in the past that in grazing collisions of heavy systems the transfer of several correlated neutron pairs could occur. However, the data for one- to sixneutron transfer all lie on an exponential curve, revealing no enhancement for 2, 4 and 6 neutron transfers over the transfer of 1,2 and 3 neutrons. [9]

The excellent performance characteristics of ATLAS have allowed the use of the accelerator for rather unusual studies, i.e. as a very sensitive spectrometer for accelerator mass spectrometry (AMS). This requires extreme stability and reproducibility of an accelerator system and excellent beam qualities. AMS with small accelerators is now routinely used for dating and tracing of lighter radioisotopes, in particular ${ }^{14} \mathrm{C}$. There are many possible applications for AMS involving heavier radioactive nuclei; in general, however, this requires a larger accelerator. The excellent machine characteristics have allowed the use of ATLAS for AMS. Measurements were successfully performed for a number of heavy radioisotopes, including $60 \mathrm{Fe}$ and $59 \mathrm{Ni}$ in meteorites and in moon rock [10], among others. Recent applications, involving the positive ion injector PII, have concentrated on noble gases which cannot be studied at the tandems widely used in AMS. Figure 12 shows as an example measurements of 39 Ar [11], potentially of imporiance for environmental studies, to concentrations as low as $8 \times 10^{-16}$ for natural samples.

A program in atomic physics takes advantage of the highly-stripped ions at ATLAS for various spectroscopic studies: Lamb-shifts in two-electron ions, two-photon transitions, precise lifetime measurements for atomic states etc. Other studies at ATLAS are directed towards material sciences and applications: columnar pinning of high $T_{c}$ superconductors for which the heavy beams at ATLAS provide maximum pinning density; wear studies with implanted radioactive ${ }^{18} \mathrm{~F}$; breakup studies of $\mathrm{C}_{60}$ molecules and other fullerines.

We discuss the latter as an example. The discovery of the highly stable and symmetric quasispherical molecule $\mathrm{C}_{60}$ and related fullerenes has led to intense studies on a wide variety of the properties of this newly found form of carbon that can now be produced in macroscopic quantities. Atomic collision techniques offer a powerful tool for investigating fullerene structures and dynamics and several such studies have already been reported. In the experiments at ATLAS the center-of-mass energies

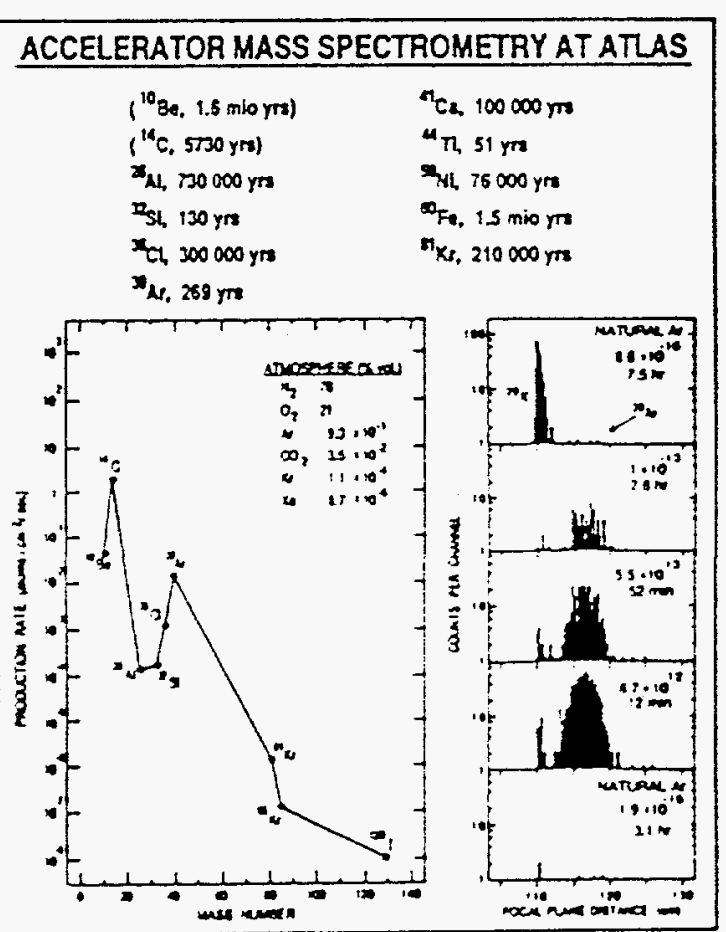

Figure 12. Accelerator mass spectrometry of 39 Ar with ATLAS. 

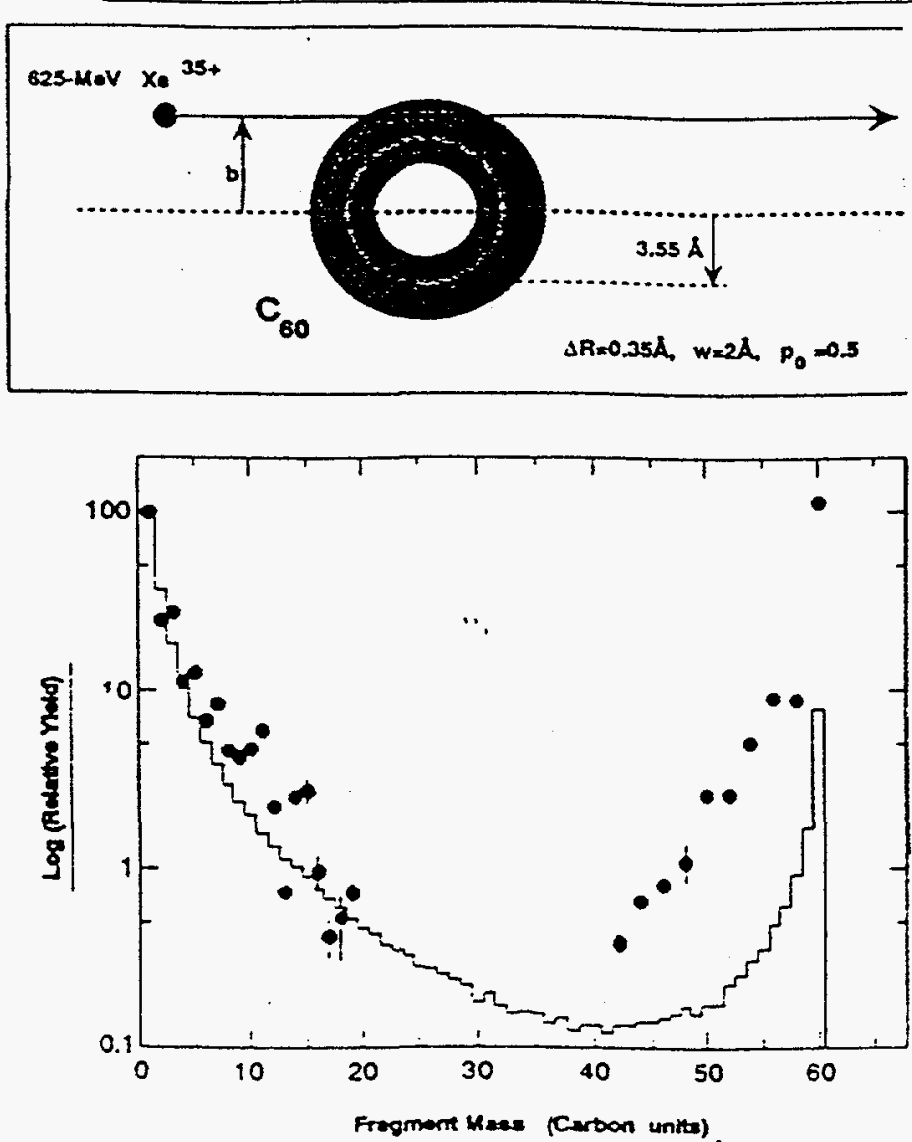

Figure 13. Measured mass distribution (solid points) and predictions of a bond-percolation model for multifragmentation of $C_{60}$ molecules by 625 $\mathrm{MeV}^{136} \mathrm{Xe}^{35+}$.

exceeded those used in previous work by several orders of magnitude. The high values of projectile velocity and charge state result in excitation and decay processes differing significantly from those seen in

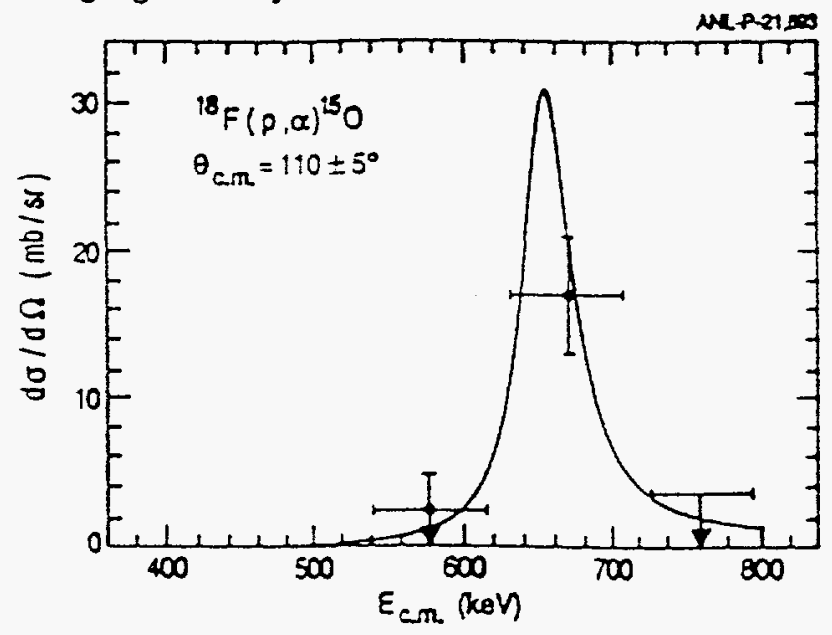

Figure 14. Cross sections for the $18 \mathrm{~F}(p, \alpha) 15_{0}$ reaction at energies of astrophysical interest. studies at lower energies. Figure 13 shows results from these studies. Plotted are mass distributions of fragments produced in the ionization and multifragmentation of $\mathrm{C}_{60} \mathrm{by}$ bombardment with $\mathrm{Xe}^{35+}$ and $\mathrm{Xe}^{18+}$ ions. The histogram is a prediction from a bondpercolation model to describe the multifragmentation processes [12].

A final example of research done at ATLAS invokes a new direction: beams of radioactive nuclei. There is large interest worldwide in extended radioactive beam capabilities, both for nuclear structure and nuclear astrophysics research. We have developed a concept for extension of ATLAS to a major ISOL type radioactive beam facility (discussed in the last section). In preparation for the research program

first experiments were carried out at ATLAS with a radioactive ${ }^{18} \mathrm{~F}$ beam [23]. The ${ }^{18} \mathrm{~F}(\mathrm{p}, \alpha)^{15} \mathrm{O}$ reaction was studied at energies which are of interest to explosive nucleosynthesis, i.e. in the laboratory energy range around $600 \mathrm{keV}$ per nucleon. Good transmission of these low-energy beams was achieved through the full
ATLAS system. The gas-filled magnet technique was used with the Enge split-pole spectrograph to achieve clean identification of the reaction products.

The results are shown in Figure 14. Cross sections were measured at three energies at an angle of $\theta_{\mathrm{cm}}=110 \pm 5^{\circ}$. The solid line corresponds to a resonance at an excitation energy of $7.066 \mathrm{MeV}$ in ${ }^{19} \mathrm{Ne}$ with a total width of $40 \mathrm{keV}$, taken from the literature. The point at $660 \mathrm{keV}$ represents the average of three measurements. The horizontal bars reflect the energy interval covered by the target thickness. The results are consistent with an swave resonance in the ${ }^{18} \mathrm{~F}(\mathrm{p}, \alpha)$ reaction that is likely to play an important role at energies of astrophysical interest.

\section{Proposal for a Radioactive Beam Facility}

The acceleration of beams of unstable nuclei has opened up new research frontiers. Experiments at existing accelerators, and particularly at the first generation of radioactive ion beam facilities, have convincingly demonstrated that unique new information becomes accessible. Critical cross sections for astrophysical processes that were impossible to obtain previously, qualitatively new and unexpected nuclear structure effects in nuclei far from stability, completely new approaches to studies of nuclear decays, reactions and structure, all have triggered much excitement for this new dimension in nuclear research. To explore this new dimension an extension of present technical capabilities and facilities is needed.

We have developed a proposal for a two-accelerator ISOL-type facility based on ATLAS [14] to provide intense beams of highest quality at the energies required for nuclear structure research and for reactions of astrophysical interest (Figure 15). Our base design uses a 


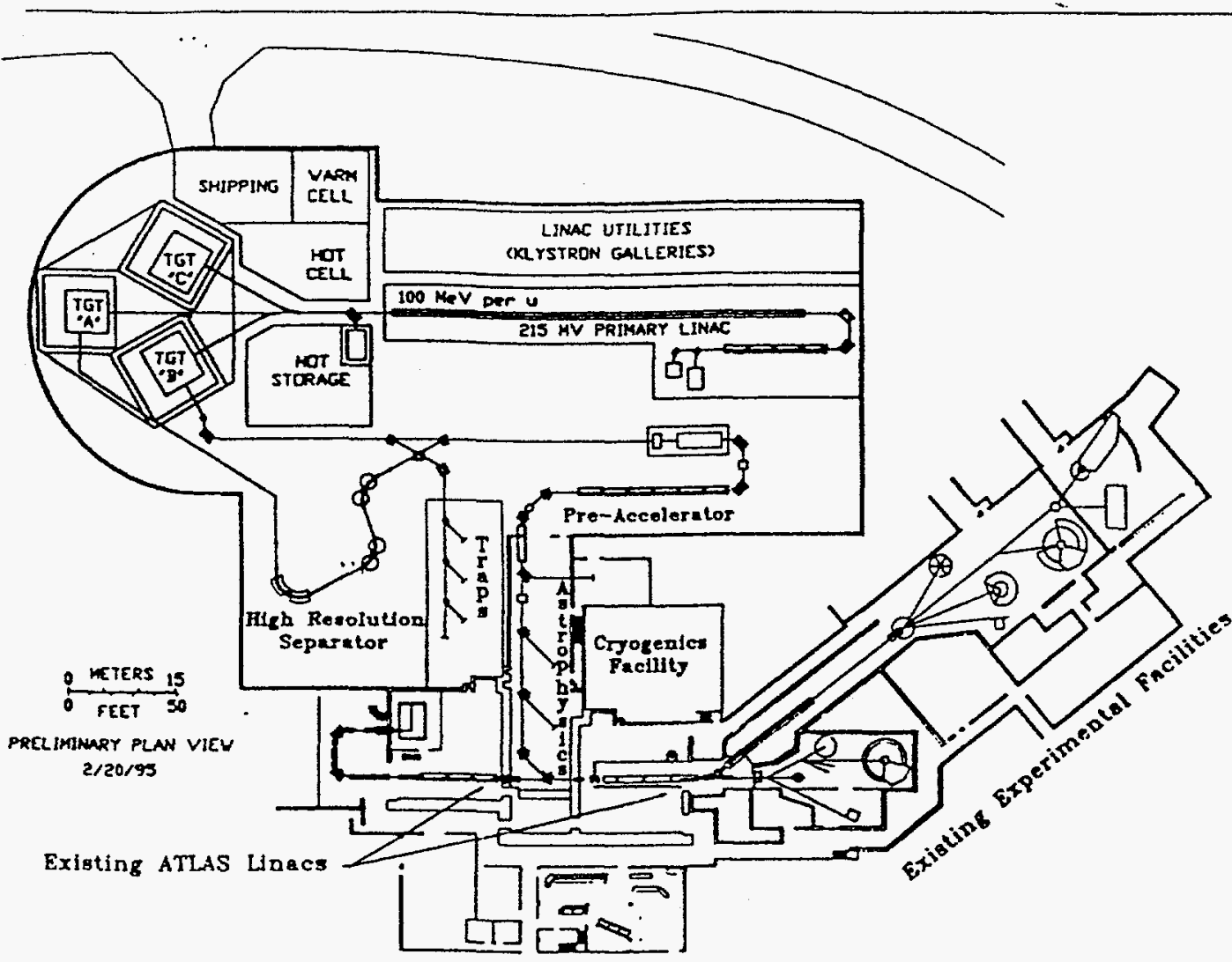

Figure 15. Layout of the proposed ISOL-type radioactive beam facility based on ATLAS.

from ISOLDE). This leads to predicted radioactive beam currents on target of, for example, $9.6 \times 10^{9}$ 132 Sn with a uranium production target.

The predicted production yields for energetic neutrons in uranium have recently been verified in an experiment [16] with $200 \mathrm{MeV}$ deuterons at the MSU National Superconducting Cyclotron Laboratory (Figure 17). Ion source configurations for the proposed facility are based on the concepts and extensive experiences gained at ISOLtype facilities (in

flexible approach for the primary accelerator and builds on the availability of the state-of-the-art heavy-ion accelerator ATLAS as the post accelerator. This is particularly important in view of the stringent beam quality requirements for nuclear structure and astrophysics experiments.

The core idea of our concept is the use of about $0.1 \mathrm{pmA}$ of 100 $\mathrm{MeV}$ neutrons from the breakup of $0.5 \mathrm{pmA}$ of $200-\mathrm{MeV}$ deuterons [15]. Spatial separation between the breakup target and the radioisotope production target solves some of the ion source problems encountered at high beam powers with charged-particle beams that undergo predominantly electronic (heat and radiation damage generating) energy loss. The purely nuclear energy loss and the long range of neutrons allow rather large target thicknesses to be used for more effective isotope production. To retain fexibility for optimizing secondary production yields by using a variety of radionuclide production mechanisms, the choice for the primary accelerator is a $215 \mathrm{MV}$ linear accelerator which can deliver a range of ions at a beam power of $100 \mathrm{~kW}$ (beam currents of $1 \mathrm{pmA}$ for protons, $0.5 \mathrm{pmA}$ for deuterons, $0.25 \mathrm{pmA}$ for alphas, $0.08 \mathrm{pmA}$ for ${ }^{12} \mathrm{C}$, and $0.06 \mathrm{pmA}$ of $18 \mathrm{O})$. Figure 16 shows predicted production yields in thick targets per particle $\mu \mathrm{C}$ (or production yields per second for beams of $(\mathrm{p} \mu \mathrm{A})$. These yields need to be multiplied with the beam intensities ( factor 100 for the neutron 'beam') and the ion source extraction efficiency (based on results particular at ISOLDE at CERN). In addition, the facility includes a high-resolution isobaric-mass separator, followed by a new low $\mathrm{q} / \mathrm{m}$,

\section{Cs Isolope production}

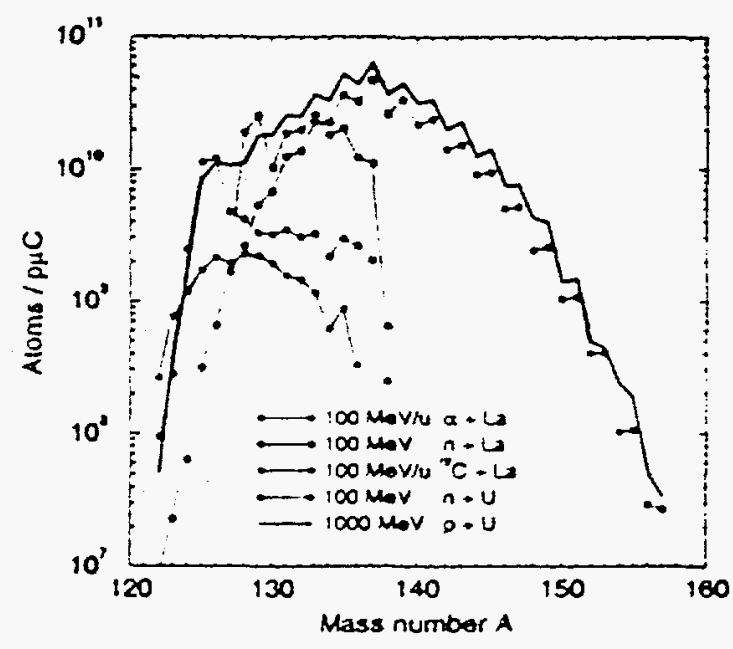

Figure 16. Production-yield predictions (per $\mu C)$ for various primury beams of an ISOL-rype radioactive heam faciliry 


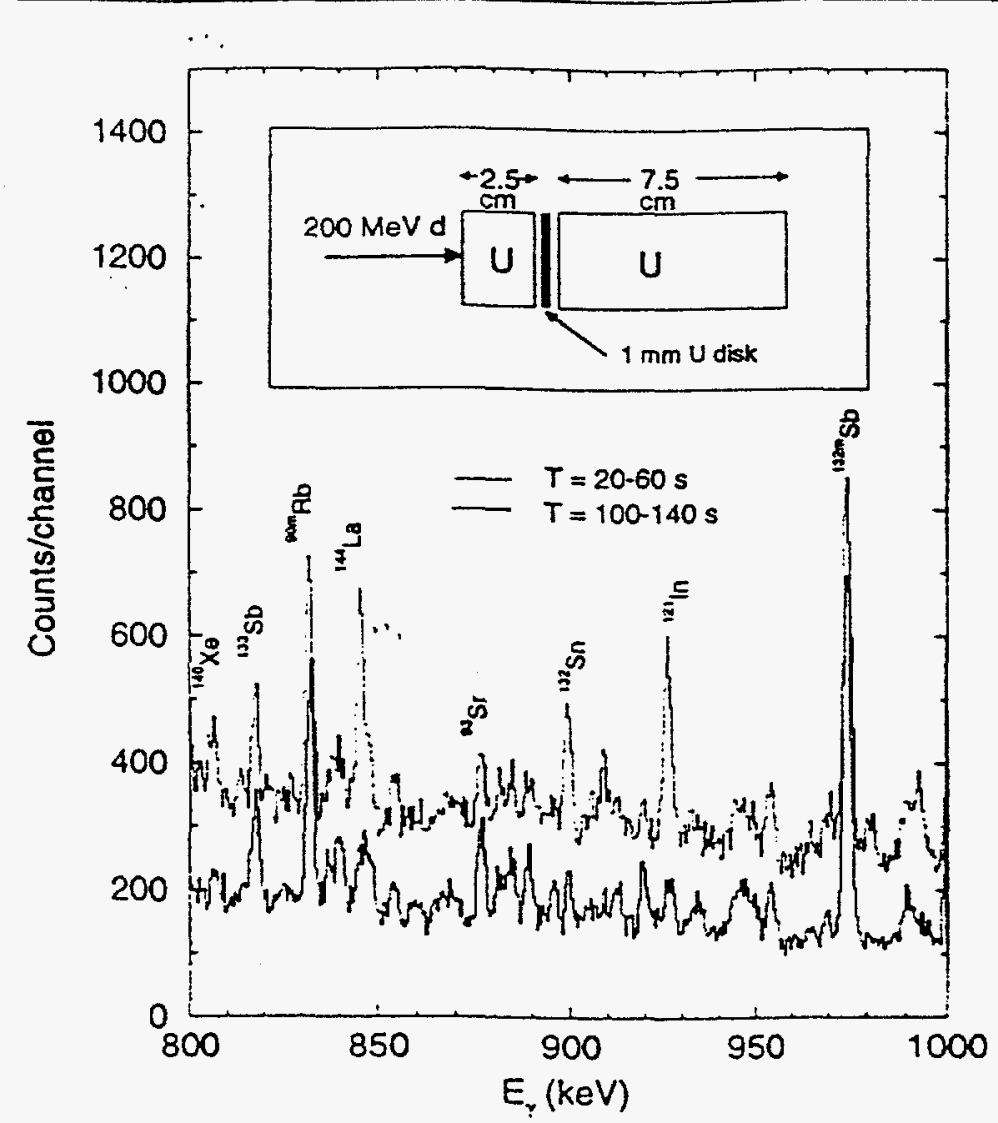

Figure 17. Setup and gamma-ray spectra measured for a determination of ${ }^{132} \mathrm{Sn}$ production with 200$\mathrm{MeV}$ deuterons incident on a uranium target.

low velocity accelerator section, $90 \%$ of which is directly based on existing ATLAS superconducting technology. The beams are then either used directly after the ion source (for research with ion traps), after low-energy acceleration up to $1 \mathrm{MeV} / \mathrm{u}$ (for experiments in nuclear astrophysics), or injected into the existing ATLAS facility for acceleration to energies above the Coulomb barrier (for studying nuclear reactions and structure).
8. 1. Ahmad et al. (APEX collaboration); submitted for publication. 9. C. L. Jiang et al., Phys. Lett. B337 (1994) 59.

10. W. Kutschera et al., Nucl. Instr. and Meth. 73B (1993) 403.

11. W. Kutschera et al., Nucl. Instr. and Meth. B92 (1994)

12. T. LeBrun et al., Phys. Rev. Lett. 72 (1994) 3965.

13. K. E. Rehm et al., submitted for publication.

14. K. E. Rehm et al., Working Paper on an Exotic Beam Facility Based on ATLAS (1995).

15. J. A. Nolen, Proc. 3rd Int. Cont. on Radioactive Nuclear Beams, East Lansing, Michigan, May 24-27, 1993; Editions Frontiers (1993) WI.

16. I. Ahmad et al., unpublished (see ref 14).
1. L. M. Bollinger, Ann. Rev. Nucl. Part. Sci. 36 (1986) 475; and references therein.

2. J. Aron et al., IEEE Trans. Nucl. Sci. NS20 (1973) 76.

3. K. W. Shepard et al., IEEE Trans. Nucl. Sci NS24 (1977) 1147.

4. L. M. Bollinger and K.W. Shepard, Proc. 1984 Linear Acc. Conf., Seeheim, Germany, May 7 11, 1984. GSI-84-11! (1984) 217; L. M. Bollinger et al., Nucl. Instr. and Meth. B328 (1993).

5. C. N. Davids et al., Nucl. Instr. and Meth. B70 (1992) 358.

6. A. M. Baxter et al., Phys. Rev. C48 (1993) 2140.

7. C. N. Davids et al., Proc. Int. Conf. on Exolic Nuclei and Alomic Masses. Arles. France. June 19-23. 1995. 